\title{
„Viel Lärm um nichts?“ \\ Fachsprachenlehrwerke im Spannungsfeld zwischen Theorie und Praxis. Eine vergleichende Analyse berufs- und fachbezogener DaF-Lehrwerke aus dem Bereich Wirtschaft ${ }^{1}$
}

Claudia Groß ${ }^{2}$

\begin{abstract}
Through a comparative analysis of DaF-textbooks ( $\mathrm{DaF}=$ Deutsch als Fremdsprache: German as a Foreign language) of "business German” and "professional German” I explore in this article as to what extent general language and special language textbooks differ from one another. This study not only highlights similarities and differences in the explored textbooks but also opens up a new perspective on the problems, which arise through the very particular requirements of special language textbooks. The outcome of my study implies that, although special language textbooks differ from general textbooks in their conceptualization, in fact their differences to general language textbooks tend to be marginal.
\end{abstract}

Keywords: DaF-didactics, textbook analysis, special language, business German, professional German

Zusammenfassung: Anhand einer vergleichenden Analyse von DaF-Lehrwerken aus dem Bereich „Wirtschaftsdeutsch“ und „Berufsdeutsch“ wird der Frage nachgegangen, inwiefern sich gemeinsprachliche und fachsprachliche Lehrwerke voneinander unterscheiden. Dabei werden nicht nur Unterschiede bzw. Gemeinsamkeiten sichtbar gemacht, sondern auch Probleme aufgezeigt, die sich aus den besonderen Anforderungen an Fachsprachenlehrwerke ergeben. Die Ergebnisse der Untersuchung weisen darauf hin, dass sich fachsprachliche Lehrwerke zumeist vor allem idealtypisch von gemeinsprachlichen unterscheiden, in der konkreten Umsetzung sind die Unterschiede bisweilen marginal.

Stichwörter: DaF-Didaktik, Lehrwerksanalyse, Fachsprachen, Wirtschaftsdeutsch, Berufsdeutsch

Resumo: A partir da análise comparativa de obras didáticas de DaF (= Deutsch als Fremdsprache: Alemão como Língua Estrangeira) das áreas de "alemão para economistas" e de "alemão para profissionais", este trabalho investiga até que ponto obras didáticas para o ensino da linguagem comum se distinguem de obras didáticas para o ensino de linguagens técnicocientíficas. Além das diferenças e das semelhanças das obras didáticas analisadas, esta pesquisa mostra também os problemas surgidos das necessidades específicas de obras didáticas para o ensino de linguagens técnico-científicas. Os resultados da pesquisa apontam que, embora existam diferenças entre a concepção das obras didáticas para o ensino da linguagem comum e aquelas para o ensino de linguagem técnico-científica, na prática, essas diferenças são insignificantes.

Palavras-chave: Didática de DaF, análise de obras didáticas, linguagens técnico-científicas, alemão para economistas, alemão para profissionais.

\footnotetext{
${ }^{1}$ Die vorliegende Untersuchung ist im Rahmen eines DaF-Hauptseminars im Wintersemester 2008/2009 an der Technischen Universität Berlin bei Prof. Dr. Maria Monteiro entstanden.

${ }^{2}$ Master-Studierende Deutsch als Fremdsprache an der Technischen Universität Berlin, E-Mail: claudia.gross.berlin@googlemail.com
} 


\section{Einleitung}

Fachsprachlicher bzw. fachbezogener Fremdsprachenunterricht gewinnt zweifelsohne an Bedeutung nicht nur in der theoretischen Diskussion, sondern vor allem in der didaktischen Praxis. Anzeichen dafür sind ein wachsendes Angebot an fachsprachlichen Kursen, Lehrmitteln und spezifischen Prüfungen.

Worin genau unterscheiden sich nun aber fachbezogene und allgemeine Fremdsprachenvermittlung? Die Beantwortung der Frage nach den Unterschieden zwischen bzw. dem Verhältnis von Fachsprache und Gemeinsprache, die HofFMANN (1985: 48) zur „Frage der Fragen in der Fachsprachenforschung“ erhob, ist dabei nicht nur eine Herausforderung für Linguisten, sondern letztlich vor allem für die Fremdsprachendidkatik von zentraler Relevanz. Angehende DaF-Lehrer werden spätestens in ihrer Ausbildung mit der Bedeutung fachbezogener Fremdsprachenvermittlung konfrontiert und stehen vor der ganz praktischen Frage, wie sie als Sprachlehrer ohne entsprechende Fachkompetenz einen fachbezogenen DaFUnterricht gestalten können. Noch stärker als im (all)gemeinsprachlichen Unterricht ${ }^{3}$ ist der unerfahrene DaF-Lehrer auf entsprechende Lehrwerke zur Vorbereitung und Durchführung seines Unterrichts angewiesen.

Von dieser Situation ausgehend, und als angehende DaF-Lehrerin selbst betroffen, habe ich mich mit der Frage befasst, was denn das „spezifisch Fach(sprach)liche“ fachbezogener DaF-Lehrwerke ausmacht, welches sie als fachsprachliche Lehrwerke qualifiziert und von gemeinsprachlichen Lehrwerken abgrenzt. Damit verbunden ist auch die Frage, ob eine strenge Abgrenzung fach- und gemeinsprachlicher Lehrwerke überhaupt sinnvoll bzw. möglich ist. Zur Beantwortung dieser Fragen habe ich eine Analyse fachsprachlicher Lehrwerke exemplarisch am Beispiel wirtschaftssprachlicher Lehrwerke durchgeführt und mit Hilfe ihrer Ergebnisse Thesen formuliert.

\section{Theoretische Grundlagen und Untersuchungsdesign}

Im folgenden Abschnitt werden einige theoretische Vorüberlegungen getroffen, sowie Ziele und Vorgehen der Analyse genauer beschrieben.

\footnotetext{
${ }^{3}$ Im Folgenden verwende ich für allgemeinen im Gegensatz zu fachsprachlichem DaF-Unterricht den Begriff ,gemeinsprachlich“. Dies entspricht der begrifflichen Setzung in der linguistischen Debatte und erlaubt eine zweifelsfreie Zuordnung.
} 


\subsection{Theoretische Vorüberlegungen}

Um die Frage nach Gemeinsamkeiten bzw. Unterschieden zwischen gemeinsprachlichen und fachsprachlichen Lehrwerken adäquat beantworten zu können, gilt es zunächst das Begriffspaar Gemeinsprache und Fachsprache zu definieren. Plausibel erscheint HofFMAnNs (1985: 53) Definition, wonach unter Fachsprache „die Gesamtheit aller sprachlichen Mittel, die in einem fachlich begrenzbaren Kommunikationsbereich verwendet werden, um die Verständigung zwischen den in diesem Bereich tätigen Menschen zu gewährleisten“ zu verstehen ist. Sehr viel schwieriger hingegen ist die Abgrenzung des Begriffes Gemeinsprache, wie aus den verschiedensten Versuchen seiner Definition als „Gesamtsprache“, „Alltagssprache“ oder auch „Standardsprache“ ersichtlich wird. ${ }^{4}$ Gemeinsprache kann nur in Gegenüberstellung zur Fachsprache definiert werden, jedoch besteht genau hierin das Dilemma: Während die Fachsprache nach o.g. Definition sich über den fachlich begrenzbaren Kommunikationsbereich, also das jeweilige Fach mit seinen spezifisch verwendeten Fachtextsorten bestimmen lässt, ist der Kommunikationsbereich der Gemeinsprache $\mathrm{zu}$ allgemein und allumfassend, als dass eine dichotomische Gegenüberstellung möglich wäre. Hinzu kommt, dass sich Fachsprache auch gemeinsprachlicher Mittel bedient, so dass sie eher einer Art Sondersprache innerhalb der Gemeinsprache entspricht. In diesem Sinne beziehe ich mich auf Hoffmanns Subsprachenmodell, in welchem er Gemeinsprache mit Gesamtsprache gleichsetzt, welche er als die „Gesamtheit aller sprachlichen Mittel“ definiert, „aus der die Subsprachen ihr Material beziehen“ (HofFMANN 1985: 50). Insofern ist jede Fachsprache als eine Subsprache der Gemeinsprache aufzufassen, deren Besonderheiten „aber nur in der Auswahl und Zusammenstellung der sprachlichen Mittel zu einem speziellen Zweck, nicht in den sprachlichen Mitteln selbst“ liegt (HoFFMANN 1985: 50).

Die explizite Bestimmung und Eingrenzung einer Fachsprache erfolgt nach Hoffmanns Modell durch die horizontale Gliederung nach den Fächern (also die Abgrenzung der Fachsprachen untereinander) sowie durch die vertikale Schichtung nach Fachlichkeitsgrad (also die Differenzierung innerhalb einer Fachsprache) (vgl. Hoffmann 1985: 58ff.). Damit rückt der Begriff der Fachlichkeit ins Zentrum der Betrachtung. Nach KALVERKÄMPER (1990: 124 apud HOFFMANN 1998: 163) ist

\footnotetext{
${ }^{4}$ Einen Überblick über die verschiedenen Auffassungen des Begriffes in der linguistischen Debatte gibt der Aufsatz von HofFmann (1998). Weitere Literaturhinweise finden sich in der Arbeit von BECKER/HUNDT (1998: 121).
} 
Fachlichkeit bzw. damit einhergehend Fachsprachlichkeit als kontunierliche Skala zu verstehen, auf der jedes Sprechen zwischen den Polen „(extrem) merkmalreich“ und „(extrem) merkmalarm“ anzusiedeln ist. FLUCK (1998: 945) bezeichnet Fachlichkeit im fachbezogenen Fremdsprachenunterricht als den „Umgang mit Fachtexten als Medium und Ziel bei der Vermittlung von (Fach-)Wissen und Kommunikationsfähigkeit im Fach.“. Im Sinne des erweiterten Fachsprachenbegriffs HofFMANNs (s.o.) versteht FLUCK (1998: 949) „Fachsprache nicht auf bestimmte morphologische, lexikalische, syntaktische oder textuelle Strukturen reduziert, sondern [...] im Sinne der modernen Fachsprachenlinguistik ganzheitlich [...] als die Sprache im Fach.“.

Diese theoretischen Vorüberlegungen dienen lediglich als Grundlage zum Verständnis des Untersuchungsdesigns meiner Analyse und ihrer Einbettung in die fachsprachenlinguistische Diskussion. Ziel der Analyse ist jedoch in erster Linie, Hinweise für die didaktische Praxis zu geben, insofern sollen die Einzelheiten der theoretischen Debatte an dieser Stelle nicht weiter erörtert werden. Für einen detaillierten Überblick empfehle ich die Arbeiten von HOFFMANN (1998) und BECKER/HundT (1998).

\subsection{Untersuchungsziele}

Die vorliegende Arbeit ist das Ergebnis einer empirischen Analyse. Grundsätzlich wurden mit der Untersuchung zweierlei Anliegen verfolgt: Einerseits sind die Ergebnisse der Analyse Grundlage für die Beantwortung der eingangs gestellten Frage nach der Besonderheit fachsprachlicher Lehrwerke im Vergleich zu gemeinsprachlichen Lehrwerken. Andererseits ist die Lehrwerksanalyse selbst auch als eine Art praktische Handreichung für DaF-Lehrer zu verstehen, die einen Überblick über vorhandene Fachsprachenlehrwerke aus dem Bereich Wirtschaft verschaffen und Anregungen für deren Einsetzbarkeit im Unterricht geben soll.

\subsection{Untersuchungsdesign}

Die Entscheidung, die Analyse von Fachsprachlehrwerken auf den Fachbereich Wirtschaft einzugrenzen, ergab sich aus ganz pragmatischen Faktoren. Erstens ist das Lehrwerksangebot in diesem Bereich recht breit, was darauf schließen lässt, dass „Wirtschaftsdeutsch“ eine wichtige Bedeutung in der DaF-Vermittlung einnimmt und 
FLUCKs (1992: 176) These bestätigt, dass „die Lehre der Wirtschaftssprachen zu einem beherrschenden Thema geworden [ist].“. Zweitens lag der Entscheidung die Annahme zu Grunde, dass Lehrwerke für „Wirtschaftsdeutsch“, im Gegensatz zu beispielsweise naturwissenschaftlichen oder technischen Fachsprachen, auch bei relativ hohem Fachlichkeitsgrad dem Laien zumindest teilweise über sein Allgemeinwissen (z.B. über Zeitungslektüre des Wirtschaftsteils) bzw. über seine Erfahrungen als Konsument von Waren und Dienstleistungen erschließbar sind. Diese Annahme hat sich im Übrigen bei meiner Analyse bestätigt.

Weiterhin habe ich mich bei der Auswahl der Lehrwerke auf jene beschränkt, die „Wirtschaftdeutsch“ als Kommunikationssprache innerhalb von oder zwischen Wirtschaftsunternehmen verstehen. Demzufolge sind (bis auf eine Ausnahme: Studium Wirtschaftsdeutsch) betriebs- oder volkswirtschaftliche Lehrwerke nicht in die Analyse eingeflossen. Aus dem gleichen Grund habe ich schließlich auch zwei „berufssprachliche“ Lehrwerke in die Analyse einbezogen, da „Deutsch im Beruf“ häufig als „ein im Sinne der ökonomischen Globalisierung modernisiertes Wirtschaftsdeutsch interpretiert wird“ (FUNK 2001: 962), wie beispielsweise an der Konzeption und den Anforderungen der Zertifikatsprüfung Deutsch für den Beruf des Goethe-Instituts $^{5}$ erkennbar ist. „Berufssprache“ Deutsch nimmt demnach eine Mittelstellung zwischen Fachsprache Wirtschaftsdeutsch und Deutsch als Fremdsprache im Sinne der Gemeinsprache ein (vgl. FunK 2001).

\subsection{Analysierte Lehrwerke}

Fachsprache Wirtschaftsdeutsch:

- Kelz, Heinrich P. / NeUf-MünKEL, Gabriele u.a. Deutsch im Beruf - Wirtschaft (Band 1, 2), Köln, Dürr + Kessler, 1994

- Volgnandt, Gabriele / VolgnANDT, Dieter / ERHARDT, Claus. Exportwege Wirtschaftsdeutsch für die Grundstufe 1. Köln, Dürr + Kessler, 1999

- Butzphal, Gerlinde / RiORDAN, Colin. Studium Wirtschaftsdeutsch. Braunschweig, Diesterweg, 1991

- Conlin, Christine. Unternehmen Deutsch. München, Klett, 1995

\footnotetext{
${ }^{5}$ Nähere Informationen zur Prüfung unter: http://www.goethe.de/lrn/prj/pba/bes/zdb/deindex.htm (08.02.2009)
} 
- Buhlmann, Rosemarie (Hg.). Wirtschaftsdeutsch Außenhandel. Warschau, POLTEXT-Verlag, 2001

- Nicolas, Gerd / MACAIRE, Dominique. Wirtschaftsdeutsch für Anfänger (Grundstufe, Aufbaustufe). München, Klett, 1995

- Eismann, Volker. Wirtschaftskommunikation Deutsch. Berlin München, Langenscheidt, 2008 (Neubearbeitung)

Berufssprache Deutsch:

- HöfFgen, Adelheid. Deutsch lernen für den Beruf. Ismaning, Hueber, 2001

- Becker, Norbert / Braunert, Jörg u.a. Dialog Beruf. Deutsch als Fremdsprache für die Grundstufe (Starter, Band 1, 2). Ismaning, Hueber, 1997

\subsection{Analysekriterien}

Dem Lehrwerksvergleich ging eine umfassende Analyse voraus, welche auf Grundlage des nachfolgend dargestellten Analyserasters durchgeführt worden ist. Die Entwicklung des Analyserasters erfolgte in Anlehnung an bestehende Kriterienkataloge ${ }^{6}$ die von mir um weitere Kriterien, die mir aus meiner didaktischen Praxis als relevant erscheinen, ergänzt worden sind. In Abbildung 1 ist das dem Lehrwerksvergleich zu Grunde liegende Analyseraster dargestellt, welches ich aus Gründen der Nachvollziehbarkeit um Anmerkungen zur Erklärung der einzelnen Kriterien ergänzt habe.

\section{Abbildung 1: Analyseraster}

\begin{tabular}{|c|c|c|}
\hline & & Erläuterungen \\
\hline & Bestandteile des Lehrwerks & $\begin{array}{l}\text { z.B. Lehrbuch, Arbeitsbuch, } \\
\text { Audiomaterial etc. }\end{array}$ \\
\hline \multirow[t]{4}{*}{ A) Zielgruppe } & Zielgruppe & $\begin{array}{l}\text { z.B. Studierende, Berufstätige im } \\
\text { Bereich Wirtschaft, } \\
\text { „Wirtschaftsinteressierte“ etc. }\end{array}$ \\
\hline & Sprachniveau & vorausgesetztes sprachliches Niveau \\
\hline & Einsatz des Lehrwerks & $\begin{array}{l}\text { kurstragend? kursbegleitend? } \\
\text { Selbststudium? }\end{array}$ \\
\hline & $\begin{array}{l}\text { Fachliche Anforderungen } \\
\text { an den Lehrer }\end{array}$ & $\begin{array}{l}\text { - z.B. Vorhandensein von } \\
\text { „Hintergrundinformationen“, }\end{array}$ \\
\hline
\end{tabular}

\footnotetext{
${ }^{6}$ Als richtungweisend im DaF-Bereich kann der Kriterienkatalog des Mannheimer Gutachtens (1977, 1979) gesehen werden. Für fachsprachliche Lehrwerke findet sich nur bei BuHLMANN (1982) ein, wie sie selbst sagt, „minimalistischer“ Kriterienkatalog zur Analyse und Bewertung fachbezogener Fremdsprachenlehrwerke, der mir als Anregung für mein eigenes Analyseraster diente.
} 


\begin{tabular}{|c|c|c|c|}
\hline & & & $\begin{array}{l}\text { Erklärung fachspezifischer Lexik } \\
\text { - bzw. entfällt, da Fachlichkeitsgrad } \\
\text { insgesamt niedrig? }\end{array}$ \\
\hline \multirow[t]{3}{*}{ B) Lernziele } & \multicolumn{2}{|c|}{ Lernzielbestimmungen } & $\begin{array}{l}\text { im Lehrwerk angegebene } \\
\text { übergeordnete Lernziele }\end{array}$ \\
\hline & \multicolumn{2}{|c|}{ Fertigkeiten } & $\begin{array}{l}\text { im Lehrwerk angegebene } \\
\text { fertigkeitsbezogene Lernziele }\end{array}$ \\
\hline & \multicolumn{2}{|c|}{ Prüfungsvorbereitung } & $\begin{array}{l}\text { im Lehrwerk angegebene } \\
\text { Vorbereitung auf eine } \\
\text { fachsprachliche Prüfung }\end{array}$ \\
\hline \multirow[t]{2}{*}{$\begin{array}{l}\text { C) Aufbau und } \\
\text { Gestaltung }\end{array}$} & \multirow[t]{2}{*}{ Aufbau } & des Lehrwerks & $\begin{array}{l}\text { z.B. Vorwort, Inhaltsübersicht, } \\
\text { Kapitelanordnung, Anhang }\end{array}$ \\
\hline & & der Lektionen & $\begin{array}{l}\text { Einteilung und Abfolge der } \\
\text { Lektionsteile }\end{array}$ \\
\hline \multirow[t]{4}{*}{ D) Inhalt } & \multicolumn{2}{|c|}{ Themen und Kommunikationssituationen } & $\begin{array}{l}\text { - fachspezifische Situationen aus } \\
\text { Wirtschaftsunternehmen? (z.B. } \\
\text { Messen, Logistik etc.) } \\
\text { - berufsspezifische Situationen? (z.B. } \\
\text { Bewerbungsgespräch, Mitarbeiter- } \\
\text { versammlung etc.) }\end{array}$ \\
\hline & \multirow[t]{2}{*}{ Textsorten } & Fachspezifisch? & $\begin{array}{l}\text { Vorhandensein von } \\
\text { wirtschaftssprachlichen (z.B. } \\
\text { Vertrag, Geschäftsbericht) oder } \\
\text { berufssprachlichen (z.B. } \\
\text { Stellenausschreibung, Visitenkarte) } \\
\text { Textsorten? }\end{array}$ \\
\hline & & Authentisch? & $\begin{array}{l}\text { - in Abgrenzung zu fiktiven Texten } \\
\text { (z.B. arbeiten einige Lehrwerke mit } \\
\text { Originaltexten - Verträgen, } \\
\text { Geschäftsberichten, Organigrammen } \\
\text { - aus Wirtschaftsunternehmen) } \\
\text { - Quellennachweise? }\end{array}$ \\
\hline & \multicolumn{2}{|c|}{ Landeskunde } & $\begin{array}{l}\text { - Inhalte: } \rightarrow \text { Berücksichtigung fach- } \\
\text { bzw. berufsspezifischer landeskund- } \\
\text { licher Informationen (z.B. Umgangs- } \\
\text { formen / Mitarbeiterhierarchien in } \\
\text { Unternehmen)? } \\
\text { - Methoden: } \rightarrow \text { kognitiv, kontrastiv, } \\
\text { interkulturell? }\end{array}$ \\
\hline $\begin{array}{l}\text { E) Methodisch- } \\
\text { didaktische } \\
\text { Konzeption }\end{array}$ & \multicolumn{2}{|c|}{ Methodisch-didaktische Konzeption } & $\begin{array}{l}\text { - Übertragbarkeit der aus der DaF- } \\
\text { Didkatik bekannten Methoden? } \\
\text { - in Anlehnung die Typologie FLUCKS } \\
\text { (1992: 190ff., 1998: 950ff.): } \\
\text { sprachorientierte, lerner- und }\end{array}$ \\
\hline
\end{tabular}




\begin{tabular}{|c|c|c|c|}
\hline & & & $\begin{array}{l}\text { fertigkeitsorientierte, textorientierte, } \\
\text { lernorientierte Ansätze }\end{array}$ \\
\hline & $\begin{array}{l}\text { Progressions- } \\
\text { prinzipien }\end{array}$ & $\begin{array}{l}\text { Grammatische } \\
\text { Progression }\end{array}$ & $\begin{array}{l}\text { - sprachsystematische Progression? } \\
\text { (grammatisch einfachere sprachliche } \\
\text { Phänomene vor komplexeren) } \\
\text { - funktionale Progression? (in der } \\
\text { Fachsprache frequentere } \\
\text { grammatische Strukturen vor weniger } \\
\text { frequenten) } \\
\text { - zyklische vs. lineare } \\
\text { Grammatikprogression }\end{array}$ \\
\hline & & $\begin{array}{l}\text { Sprachliche } \\
\text { (Fertigkeits-) } \\
\text { Progression }\end{array}$ & $\begin{array}{l}\text { - fertigkeitsspezifisch vs. } \\
\text { fertigkeitsintegrierend } \\
\text { - „Hierarchie“ der Fertigkeiten? }\end{array}$ \\
\hline & & $\begin{array}{l}\text { Fachliche } \\
\text { Progression }\end{array}$ & $\begin{array}{l}\text { - im Sinne eines ansteigenden } \\
\text { Fachlichkeitsgrads } \\
\text { - zyklisch vs. linear }\end{array}$ \\
\hline & Übungsmaterial & & $\begin{array}{l}\text { - Übungstypen (geschlossen vs. offen, } \\
\text { reproduktiv vs. produktiv, gesteuert } \\
\text { vs. ungesteuert) } \\
\text { - handlungsorientiert? (z.B. } \\
\text { kommunikativ-kooperative Aufgaben, } \\
\text { problemlösungsorientierte Aufgaben, } \\
\text { Projektaufgaben etc.) }\end{array}$ \\
\hline & Lösungsschlüsse & & vorhanden? \\
\hline & Vermittlung von & trategien? & $\begin{array}{l}\text { im Sinne eines lernerzentrierten } \\
\text { Ansatzes, d.h. Befähigung des } \\
\text { Lerners zum eigenständigen } \\
\text { vertiefenden Lernen („Lernen } \\
\text { lernen“) }\end{array}$ \\
\hline & $\begin{array}{l}\text { Vermittlung von } \\
\text { „Schlüsselqualifi }\end{array}$ & len“? & $\begin{array}{l}\text { - z.B. Teamfähigkeit, Abstraktions- } \\
\text { fähigkeit, Problemlösungs- } \\
\text { orientierung etc. } \\
\text { - Vermittlung nur durch } \\
\text { Thematisierung oder auch } \\
\text { Umsetzung durch entsprechende } \\
\text { Übungs- und Sozialformen? }\end{array}$ \\
\hline F) Bewertung & $\begin{array}{l}\text { Angemessenheit } \\
\text { bezüglich }\end{array}$ & der Zielgruppe & $\begin{array}{l}\text { z.B. hinsichtlich des vorausgesetzten } \\
\text { fremdsprachlichen Niveaus }\end{array}$ \\
\hline & & der Lernziele & $\begin{array}{l}\text { immanente Bewertung gemessen an } \\
\text { den im Lehrwerk formulierten und } \\
\text { verfolgten Lernzielen }\end{array}$ \\
\hline
\end{tabular}




\section{Ergebnisse des Lehrwerkvergleichs}

In den folgenden Abschnitten werden die Ergebnisse der Analyse in einer vergleichenden überblicksartigen Darstellung zusammengefasst. Die diesem Überblick zugrunde liegenden Einzelergebnisse wurden durch die Analyse jedes einzelnen Lehrwerks hinsichtlich der oben genannten Kriterien gewonnen.. Sie sollen an dieser Stelle nicht im Einzelnen dargestellt werden. Die zusammenfassende Darstellung soll vielmehr einen Überblick über die untersuchten Lehrwerke bieten und Tendenzen bzw. Auffälligkeiten aufzeigen.

\subsection{Bestandteile der Lehrwerke}

Es ist auffällig, dass die untersuchten Lehrwerke für Wirtschafts- und Berufsdeutsch sich in ihren Bestandteilen stärker unterscheiden als gemeinsprachliche Lehrwerke.

Der Standard eines Medienverbunds aus Lehrbuch, Arbeitsbuch, Lehrerhandreichung und Audiomaterial, wie er bei gemeinsprachlichen Lehrwerken mittlerweile üblich ist, wird bei weitem nicht von allen untersuchten Lehrwerken erfüllt. Bei zwei der untersuchten Lehrwerke (Studium Wirtschaftsdeutsch, Wirtschaftsdeutsch Außenhandel) gibt es außer dem Lehrbuch keine weiteren Lehrwerkskomponenten. Nur vier der neun untersuchten Lehrwerke (Deutsch im Beruf Wirtschaft, Wirtschaftsdeutsch für Anfänger, Wirtschaftskommunikation Deutsch, Dialog Beruf) bieten eine Lehrerhandreichung. Dieses reduzierte Angebot an Lehrwerkskomponenten ist sicherlich zum Teil dem anvisierten Lernziel (vgl. 3.3) bzw. dem vorausgesetzten fremdsprachlichen Niveau der Lerner (vgl. 3.2) geschuldet: Zum Beispiel kann ein Lehrwerk, welches in erster Linie das Verstehen geschriebener fachsprachlicher Texte schulen will, auf Audiomaterial ohne Weiteres verzichten. Ebenso ist ein Arbeitsbuch mit überwiegend grammatischen Strukturübungen nicht zwingend notwendig, wenn bereits ein fortgeschrittenes sprachliches Niveau vorausgesetzt wird. Allerdings ist vermutlich auch der These BuHLMANNs (1998: 982f.) zuzustimmen, dass hierbei auch verlagsökonomische Argumente eine Rolle spielen. Den beschränkteren Einsatzmöglichkeiten fachsprachlicher Lehrwerke und ihrer daher geringeren Auflage stehen hohe Kosten der Lehrwerksentwicklung und -produktion gegenüber. Das Ergebnis dieser Kosten-Nutzen-Rechnung spiegelt sich in einer minimalistischeren Konzeption der Lehrwerke wider. Dieses Dilemma führt meines Erachtens zu nicht 
unproblematischen Konsequenzen: Mehr noch als bei gemeinsprachlichen Lehrwerken ist eine Lehrerhandreichung für Fachsprachenlehrwerke nicht nur hilfreich, sondern zum Teil gar unabdingbar. Der Sprachlehrer ist im Normalfall fachlicher Laie und somit auf Erläuterungen der fachlichen Inhalte wie auch auf didaktische Hinweise zur Vermittlung der fachsprachlichen Strukturen angewiesen. Wenngleich dem DaF-Lehrer ein Großteil der fachspezifischen Lexik aus dem Wirtschaftsbereich über sein Allgemeinwissen bzw. seinen lebensweltlichen Alltag bekannt oder über den Kontext erschließbar sein dürften, so sind jedoch Kenntnisse über Strukturen und Prozesse wirtschaftlichen Handelns nicht ohne weiteres vorauszusetzen. Hier könnten erklärende Hinweise im Lehrerhandbuch die Diskrepanz zwischen Sprach- und Fachwissen überwinden.

\subsection{Zielgruppe und Einsatzmöglichkeiten der Lehrwerke}

Hinsichtlich der anvisierten Zielgruppen unterscheiden sich die Lehrwerke beträchtlich. Dieses Ergebnis ist allerdings u.a. auch durch die von mir getroffene Auswahl der Lehrwerke beeinflusst worden, da ich auf diese Weise eine breitere Vergleichsspanne ermöglichen konnte. Die Zielgruppenbeschreibung variiert zwischen recht allgemein (Deutsch im Beruf - Wirtschaft: Erwachsene und Jugendliche mit Interesse an deutschem Wirtschaftsleben) und besonders eng gefasst (Studium Wirtschaftsdeutsch: englische Studierende ohne Vorkenntnisse in Betriebs- oder Volkswirtschaft). Diese Beobachtung bestätigt die Tatsache, dass im Bereich der Fachsprachendidaktik die Abgrenzungskriterien von Fach- zu Gemeinsprache umstritten sind und somit auch keine Einigkeit besteht über die definitorische Eingrenzung ihrer Anwender. ${ }^{7}$

In Bezug auf das fremdsprachliche Niveau setzen fünf von neun Lehrwerken mindestens mittlere Deutschkenntnisse (etwa ab Niveau B1/B2 des Europäischen Referenzrahmens) voraus, wobei darauf hinzuweisen ist, dass einzig das Lehrwerk Wirtschaftskommunikation Deutsch explizit mit den Niveaustufen des Europäischen Referenzrahmens arbeitet. Bei allen anderen Lehrwerken geschah die Einordnung der

\footnotetext{
${ }^{7}$ Zur Bestätigung dieser These genügt ein Blick in das Inhaltsverzeichnis des Internationalen Handbuchs Fachsprachen - Languages for Special Purposes, Band 1, Abschnitt II: Auffassungen vom Status der Fachsprachen (HOFFMANN / KALVERKÄMPER / WIEGAND 1998). Einen Überblick über die Diskussion geben außerdem die bereits erwähnten Aufsätze von BECKER/HunDT (1998) im gleichen Band und von HOFFMANN (2001).
} 
sprachlichen Voraussetzungen mittelbar über andere Angaben (z.B. vorausgesetzte Unterrichtsstunden oder abgelegte Prüfungen). Weiterhin weise ich darauf hin, dass das Analyseergebnis (etwa 50-50-Verteilung von Anfänger- und Fortgeschrittenenlehrwerken) nicht unbedingt repräsentativ für den Lehrwerksmarkt im Bereich Wirtschaftsdeutsch ist, da ich bei der Auswahl der Lehrwerke Anfängerlehrwerke (Deutsch im Beruf - Wirtschaft, Exportwege - Wirtschaftsdeutsch für die Grundstufe 1, Wirtschaftsdeutsch für Anfänger, Dialog Beruf) gezielt mit einbezogen habe. Im vorhandenen Lehrwerksangebot lässt sich hingegen eine sehr starke Tendenz zu Fortgeschrittenen-Lehrwerken feststellen, da bis vor wenigen Jahren in der Fachsprachendidaktik ein Konsens darüber bestand, dass fachsprachliches Wissen erst ab einem fundierten fremdsprachlichen Niveau vermittelbar sei (vgl. BUHLMANN 1998: 986; FLUCK 1992: 109; zu berufsbezogener Fremdsprachenvermittlung vgl. FuNK 2001: 964).

Die breite Mehrheit von acht Lehrwerken ist primär für den Kursunterricht konzipiert, wovon zwei Lehrwerke Hinweise für die Verwendung zum Selbststudium geben (Deutsch im Beruf - Wirtschaft, Wirtschaftsdeutsch für Anfänger). Nur ein Lehrwerk ist zum Selbststudium angelegt und nur bedingt kursbegleitend einsetzbar (Wirtschaftsdeutsch Außenhandel).

\subsection{Lernziele}

Wie auch die Zielgruppenbestimmungen variieren die Lernzielbestimmungen zwischen sehr allgemein (Dialog Beruf Starter: Vermittlung der Grundlagen sprachlicher Handlungsfähigkeit in Alltag und Beruf) und recht explizit. Das Lehrwerk Exportwege, Wirtschaftsdeutsch für die Grundstufe 1 enthält überhaupt keine Angaben über die angestrebten Lernziele. Die meisten wirtschaftssprachlichen Lehrwerke nennen zumindest implizit kommunikative Handlungsfähigkeit im Kontakt mit deutschen Geschäftspartnern oder bei einem geschäftlichen Aufenthalt in Deutschland als primäres Lernziel, wobei Kommunikation vorrangig als mündliche verstanden wird. In diesen Lehrwerken findet sich dementsprechend meist ein fertigkeitsintegrierender Ansatz, dem eine Hierarchie der zu vermittelnden sprachlichen Fertigkeiten von Hören über Lesen zu Sprechen und erst sehr nachrangig Schreiben zu Grunde liegt. Nur ein einziges Lehrwerk kann als fertigkeitsspezifisch bezeichnet werden: Wirtschaftsdeutsch Außenhandel. Hier finden sich keine fertigkeitsintegrierenden Übungen oder Aufgaben, 
sondern je nach Lehrwerksteil werden gezielt Leseverstehen fachsprachlicher Texte, Fachterminologie und schriftliche Produktion geschult. Wie zu erwarten war, lässt sich insgesamt feststellen, dass in Anfängerlehrwerken und berufssprachlichen Lehrwerken die Lernzielbeschreibungen tendenziell breiter gefasst sind und ein größeres Gleichgewicht zwischen den einzelnen zu vermittelnden Fertigkeiten besteht.

Ein weiteres Kriterium, das ich unter dem Aspekt der Lernzielbestimmungen in meine Analyse aufgenommen habe, ist das der Prüfungsvorbereitung. Vier von neun Lehrwerken geben explizit die Vorbereitung auf eine bestimmte fachsprachliche oder berufssprachliche Prüfung an (Exportwege - Wirtschaftsdeutsch für die Grundstufe, Deutsch lernen für den Beruf, Dialog Beruf $\rightarrow$ Zertifikat Deutsch bzw. Zertifikat Deutsch für den Beruf; Wirtschaftskommunikation Deutsch $\rightarrow$ Prüfung Wirtschaftsdeutsch International). Entsprechend finden sich in diesen Lehrwerken prüfungsrelevante und -spezifische Aufgabentypen bzw. Strukturen.

\subsection{Aufbau und Gestaltung}

Hinsichtlich Aufbau und Gestaltung lässt sich feststellen, dass die Mehrzahl der untersuchten Lehrwerke sich kaum von gemeinsprachlichen unterscheidet. Es finden sich thematische Lektionen, die Lesetexte und Übungen zu (fach-)sprachlichen Mitteln und gegebenenfalls grammatischen Strukturen beinhalten und die meist nach einer linearen fachlichen und sprachlichen Progression angeordnet sind. Zusätzlich finden sich häufig im Vorwort oder Anhang Quellennachweise, Register oder Glossare. Drei Lehrwerke weichen von dieser Struktur ab. In Studium Wirtschaftsdeutsch sind die Lektionen nach Themen unterteilt, weisen jedoch keinen einheitlichen strukturellen Aufbau auf. Wirtschaftsdeutsch Außenhandel ordnet die Kapitel nach Fertigkeiten (Lexik, Textrezeption, Strukturübungen, schriftliche Produktion). Wirtschaftskommunikation Deutsch besteht aus sieben thematischen Einheiten, die in jeweils zwei bis drei Kapitel unterteilt sind. Dabei dienen die „grünen Kapitel“ der Hintergrundinformation, während die „blauen Kapitel“ fachspezifische Handlungsketten aus der Unternehmenspraxis repräsentieren. 


\title{
3.5 Inhalte
}

In Bezug auf die Inhalte (Themenwahl, Kommunikationssituationen, Fachlichkeitsgrad der Texte und Aufgaben) unterscheiden sich die untersuchten Lehrwerke erst bei näherem Hinsehen. In nahezu allen Lehrwerken finden sich Standardthemen und -situationen aus der wirtschaftlichen bzw. beruflichen Kommunikation, wie Berufs- und Unternehmensprofile, Messen, Auftragsabwicklung, Werbung, Marketing, Verkauf, Vertrieb, Logistik etc. Entsprechend weisen die meisten der Lehrwerke fach- (bzw. berufs-)spezifische Textsorten, wie Stellen- und Werbeanzeigen, Geschäftsbriefe, Rechnungen, Lieferscheine, Visitenkarten usw. auf. Das Fachlichkeitsniveau ${ }^{8}$ der Texte variiert dabei erheblich, wobei allerdings nicht immer eine Korrelation zwischen Fachlichkeitsgrad und sprachlichem Niveau des Lehrwerks besteht, wie anzunehmen wäre. Beispielhaft zu nennen sind hier die Anfängerlehrwerke Deutsch im Beruf - Wirtschaft und Wirtschaftsdeutsch für Anfänger, wobei ersteres von Anbeginn mit fachspezifischen Textsorten (auf geringerem sprachlichem Niveau) arbeitet, während letzteres sich überhaupt nicht fachspezifischer Texte bedient und sich nur in der Themenwahl nicht aber im Fachlichkeitsgrad von gemeinsprachlichen Lehrwerken unterscheidet. Das Lehrwerk Wirtschaftsdeutsch für Anfänger dient meiner Einschätzung nach als Paradebeispiel für FUNKs These:

\begin{abstract}
Wenn in berufsbezogenen Lehrwerken für Anfänger in den ersten Lektionen Situationsvorgaben wie Geschäftsbesuche und Verhandlungen gemacht werden, so ignoriert diese Vorgabe die komplexen Kommunikationsbedingungen solcher Situationen völlig und bleibt folgenlose Sprachspielerei bzw. Lernertäuschung. (FUNK 2001: 964)
\end{abstract}

In allen Lehrwerken sind die fachlichen Anforderungen an den Lehrer relativ gering. Fachsprachliche Lexik und Fachtexte sind meist über das Allgemeinwissen (Erfahrung als Konsument) oder aus dem Kontext erschließbar, einige Lehrwerke geben darüber hinaus im Lehrerhandbuch oder im Lehrbuch selbst Erklärungen zu fachlichen Hintergründen (Wirtschaftskommunikation Deutsch, Dialog Beruf, Wirtschaftsdeutsch Außenhandel, Wirtschaftsdeutsch für Anfänger). Acht von neun

\footnotetext{
${ }^{8}$ Fachlichkeit bezieht sich wie unter 2.1 dargestellt in erster Linie auf den Umgang mit Fachtexten als Medium und Ziel bei der Vermittlung von (Fach-)Wissen und Kommunikationsfähigkeit im Fach. Insofern spreche ich von einem niedrigeren fachlichen Niveau oder Fachlichkeitsgrad eines Lehrwerks, wenn die darin verwendeten Texte nicht dazu geeignet sind, dieses spezifische Fachwissen zu vermitteln bzw. auf fachspezifische Kommunikationsstrukturen verzichten.
} 
Lehrwerken geben an, mit authentischen Texten zu arbeiten, wobei allerdings nicht immer die Quellen eindeutig angegeben sind (so bei Deutsch im Beruf - Wirtschaft, Studium Wirtschaftsdeutsch, Unternehmen Deutsch). Nur das Lehrwerk Dialog Beruf scheint auf authentische Texte ganz zu verzichten.

Neben fachspezifischen Themen und Textsorten finden sich in den meisten (auch Fortgeschrittenen-) Lehrwerken (Ausnahmen: Studium Wirtschaftsdeutsch, Wirtschaftsdeutsch Außenhandel, Wirtschaftskommunikation Deutsch) auch gemeinsprachliche Kommunikationssituationen und entsprechende Textsorten, wie z.B. Essen gehen, öffentliche Verkehrsmittel etc. Nahe liegend, aber keineswegs irrelevant, ist die Feststellung, dass Anfängerlehrwerke und berufssprachliche Lehrwerke einen größeren Anteil an gemeinsprachlichen Themen und Texten und meist - wie schon erwähnt - einen geringeren Fachlichkeitsgrad haben als fachspezifische Lehrwerke für Fortgeschrittene.

Landeskundliche Informationen sind in allen Lehrwerken mindestens implizit über Texte enthalten, wobei allerdings nur drei Lehrwerke fach- oder berufsrelevante landeskundliche Informationen explizit vermitteln (Deutsch im Beruf - Wirtschaft, Wirtschaftskommunikation Deutsch, Deutsch lernen für den Beruf). Nur ein einziges Lehrwerk verfolgt dabei einen interkulturellen Ansatz (Wirtschaftskommunikation Deutsch), alle anderen beschränken sich auf kognitive bzw. kontrastive (Dialog Beruf, Unternehmen Deutsch) Landeskundevermittlung.

Ein weiterer Aspekt, den man sowohl unter inhaltlichen als auch methodischdidaktischen Gesichtspunkten (vgl. 3.6.) untersuchen kann, ist die Frage nach der Vermittlung so genannter beruflicher „Schlüsselqualifikationen“ oder „soft skills“. Hierbei handelt es sich um im Berufsleben relevante Fähigkeiten bzw. Fertigkeiten, wie z.B. Teamfähigkeit, Selbstständigkeit, Abstraktionsfähigkeit, Entscheidungs- und Problemlösungsfähigkeit, Gesprächsführung, aber auch Umgangsformen im Unternehmen oder „small talk“. Dieses Kriterium habe ich in meine Analyse aufgenommen, da Umfragen in Unternehmen und die Analyse von Stellenangeboten ergeben, dass neben (bzw. sogar noch vor) fachlichen und sprachlichen Kompetenzen vor allem die „Schlüsselqualifikationen“ als entscheidend für erfolgreiches berufliches Handeln gesehen werden. ${ }^{9}$ Da fachsprachliche Lehrwerke zumindest idealtypisch nicht

\footnotetext{
${ }^{9}$ Zur Bedeutung von „Schlüsselqualifikationen“ im berufsbezogenen Fremdsprachenunterricht vgl. FUNK (2001: 965ff.), im fachbezogenen Fremdsprachenunterricht vgl. FLUCK (1992: 176ff.)
} 
nur Sprach- sondern auch Fachlehrwerke sind, halte ich die Integration solcher „soft skills“ in die Lehrwerke für nahe liegend. Bei den untersuchten Lehrwerken finden sich nur zwei (Wirtschaftskommunikation Deutsch, in Ansätzen auch Studium Wirtschaftsdeutsch), die die Vermittlung solcher „Schlüsselqualifikationen“ nicht nur explizit konzeptionell anstreben, sondern auch durch entsprechende Aufgaben- und Sozialformen umsetzen. In vier weiteren Lehrwerken (Deutsch im Beruf - Wirtschaft, Unternehmen Deutsch, Deutsch lernen für den Beruf, Dialog Beruf) werden „soft skills“ zumindest thematisiert und zum Teil mittels Beispielen präsentiert.

\subsection{Methodisch-didaktische Konzeption}

Die Frage nach der methodisch-didaktischen Konzeption der untersuchten Lehrwerke ist nicht ohne weiteres zu beantworten, unter anderem, da die aus der allgemeinen Fremdsprachendidaktik bekannten methodischen Ansätze (GrammatikÜbersetzungsmethode, audio-visuelle/-linguale Methode, kommunikative Methode, interkulturelle Methode) nicht eins zu eins auf die Fachsprachenvermittlung übertragbar sind (vgl. BuHLmann 1998: 983f.). Hilfreich ist es, hier die Typologie von FLuCK (1992: 190ff., 1998: 950ff.) ergänzend hinzuziehen, in der (1) sprach-, (2) lerner- und fertigkeits-, (3) text- und (4) lernorientierte Ansätze unterschieden werden.

Die Mehrheit der Lehrwerke verfolgt einen kommunikativen, handlungs- und fertigkeitsorientierten Ansatz. Ausnahmen bilden dabei die Lehrwerke Wirtschaftsdeutsch Außenhandel und Wirtschaftsdeutsch für Anfänger. Ersteres folgt einer stärker textorientierten Konzeption, indem es das Verstehen von Fachtexten und Fachlexik als Grundlage und Ziel des Lernprozesses versteht. Letzteres gibt zwar an, einen kommunikativ-pragmatischen und gar interkulturellen Ansatz zu verfolgen, stellt sich aber nach der Analyse des Übungsmaterials als wenig kommunikativ heraus. Methodisch ist es am ehesten dem audio-lingualen bzw. -visuellen Ansatz zuzuordnen. Vor allem die Anfängerlehrwerke sind im Sinne FLUCKs auch als sprachorientiert zu verstehen, da sie häufig eine funktionale grammatische Progression aufweisen. Fachsprachlich relevante bzw. besonders frequente grammatische Strukturen (z.B. Passiv, Nominalisierung) werden abweichend von gemeinsprachlichen Lehrwerken besonders früh eingeführt. Umgekehrt ist auffällig, dass als weniger relevant gesehene Strukturen, wie zum Beispiel die Personalpronomen ,du' und ,ihr' und die entsprechenden Verbkonjugationen oft erst sehr spät (Wirtschaftsdeutsch für Anfänger) 
thematisiert werden. Soweit diese Konzeption nachvollziehbar erscheint, so muss dennoch darauf hingewiesen werden, dass sie aus sprachsystematischer Perspektive nicht unproblematisch ist, da grammatische Strukturen zum Teil unvollständig oder von den üblichen Bezeichnungen abweichend dargestellt werden.

Einige Lehrwerke (Deutsch im Beruf - Wirtschaft, Studium Wirtschaftsdeutsch, Unternehmen Deutsch, Wirtschaftsdeutsch Außenhandel, Deutsch lernen für den Beruf) weisen darüber hinaus auch - im Sinne FLUCKs - lernorientierte Elemente auf, indem sie explizit Lernstrategien z.B. zur Textrezeption und -produktion oder Wortschatzsemantisierung und -memorisierung vermitteln.

Hinsichtlich der Progressionsprinzipien ist, wie bereits erwähnt, häufig der funktionale Aspekt ausschlaggebend. Weiterhin wird vor allem in handlungs- und problemlösungsorientierten Lehrwerken (Exportwege - Wirtschaftsdeutsch für die Grundstufe 1, Wirtschaftskommunikation Deutsch, Unternehmen Deutsch) wie auch im Lehrwerk Wirtschaftsdeutsch Außenhandel eine steuerungsbedingte Progression der Aufgaben, im Sinne einer abnehmenden Steuerung (geschlossene Übung $\rightarrow$ offene Übung $\rightarrow$ Aufgabe) sichtbar.

\subsection{Bewertung: Angemessenheit der Lehrwerke}

Die Bewertung der Angemessenheit der Lehrwerke kann nur immanent, das heißt gemessen an den eigens gesetzten Ansprüchen, und auch nur theoretisch betrieben werden, da der Praxistest noch aussteht. Angemessenheit kann sich auf mindestens zwei Aspekte beziehen: die Zielgruppe und die Lernzielbestimmungen.

Die große Mehrheit der untersuchten Lehrwerke erfüllt im Allgemeinen mehr oder weniger die eigens gesetzten Ansprüche hinsichtlich Lernziel und Zielgruppe. Einzig beim Lehrwerk Wirtschaftsdeutsch für Anfänger ist eine deutliche Diskrepanz zwischen Lernzielformulierungen und ihrer Umsetzung festzustellen. Dem primären Lernziel der mündlichen Kommunikations- und Handlungsfähigkeit im berufs- und alltagssprachlichen Umfeld wird im Übungsapparat kaum Rechnung getragen. Die Übungen sind fast ausschließlich geschlossen-reproduktiv und mündliche Kommunikation wird hier nur durch Imitation und „starre“ Variation von vorgegebenen Dialogen geübt. Beim Lehrwerk Unternehmen Deutsch ist die Beurteilung schwierig, da es keine expliziten Angaben zum Lernziel enthält, an denen es gemessen werden könnte. Wirtschaftsdeutsch Außenhandel nennt als primäres Lernziel die sprachliche 
Handlungsfähigkeit im Fach, im Sinne der Fähigkeit, sich in der Zielsprache zu informieren und $\mathrm{zu}$ verständigen. Obwohl sprachliche Verständigung mit Kommunikationsfähigkeit gleichzusetzen ist, fehlen in diesem Lehrwerk kommunikative Aufgaben und die mündliche Sprachproduktion bleibt insgesamt vernachlässigt. Allerdings kann man hier möglicherweise dennoch davon ausgehen, dass das Lehrwerk hinsichtlich der anvisierten Zielgruppe angemessen ist, da Sprachkenntnisse auf einem recht hohen Niveau und insofern wohl auch ein bestimmtes $\mathrm{Maß}$ an mündlicher Kommunikationsfähigkeit vorausgesetzt werden.

Letztlich kann dieser Überblick nur ein exemplarischer sein. Im Einzelfall muss jedes Lehrwerk seine Einsetzbarkeit in der Praxis beweisen.

\section{Fazit: Schlussfolgerungen für die Fragestellung}

Was nun aber sagen uns die dargestellten Ergebnisse des Lehrwerksvergleichs zur eingangs gestellten Frage? Worin liegen die zentralen Unterschiede zwischen gemeinsprachlichen und fachsprachlichen Lehrwerken? Einige Antworten konnten gefunden werden. Ich möchte sie an dieser Stelle als Thesen zusammenfassen. Ich weise nochmals darauf hin, dass sich die Ergebnisse auf wirtschafts- (bzw. berufs-)sprachliche Lehrwerke beziehen und insofern nicht ohne weiteres verallgemeinerbar sind. Eine Untersuchung anderer Fachsprachenlehrwerke kann möglicherweise zu völlig anderen Ergebnissen führen.

\section{Thesen:}

1) Gemeinsprachliche und fachsprachliche Lehrwerke aus dem Bereich Wirtschaft unterscheiden sich vor allem hinsichtlich der Fachlichkeit bzw. Fachbezogenheit der ausgewählten und beschriebenen Sprachhandlungssituationen und Textsorten.

2) Berufssprachliche Lehrwerke und Anfängerlehrwerke haben einen niedrigeren Fachlichkeitsgrad. Sie unterscheiden sich inhaltlich von gemeinsprachlichen Lehrwerken in erster Linie in den Themenbereichen und Kommunikationssituationen.

3) Fachsprachliche Lehrwerke bedienen sich meist einer Mischung verschiedener didaktischer Methoden. Sie sind stärker adressatenorientiert und ihre Progression richtet sich eher nach funktionalen als formal-sprachlichen Kriterien.

4) Idealtypisch vermitteln Fachsprachenlehrwerke neben fachlichen und sprachlichen auch interkulturelle Kompetenzen und berufliche „Schlüsselqualifikationen“. Das Übungs- und Aufgabenmaterial dient nicht nur der Aneignung und Erweiterung (fach-)sprachlicher Handlungsfähigkeit, 
sondern zielt auch auf die Stärkung problemlösungsorientierten beruflichen Handelns ab.

5) Aufgrund verlagsökonomischer Argumente sind die Zielgruppen von Fachsprachenlehrwerken im Bereich Wirtschaft oft (zu) weit gefasst. Dementsprechend sinkt der Fachlichkeits- und Spezialisierungsgrad des Lehrwerkes.

Insgesamt lassen sich zwei Tendenzen feststellen: Erstens sind fachsprachliche Lehrwerke im Bereich Wirtschaft nicht als homogene Gruppe aufzufassen, da sie untereinander deutliche Unterschiede aufweisen. Zweitens unterscheiden sich wirtschaftssprachliche Lehrwerke von gemeinsprachlichen vor allem idealtypisch, in der Realität sind die Unterschiede zum Teil nur punktuell. Insbesondere die Tendenz zu verstärkter Adressatenorientierung im Lehrwerkssektor vermag dazu führen, dass künftig auch in gemeinsprachlichen Erwachsenenlehrwerken Themen und Kommunikationssituationen aus dem Berufsleben vermehrt integriert werden. ${ }^{10}$ Die Abgrenzung zu wirtschaftlichen Fachsprachenlehrwerken wird damit weiter erschwert. Letztlich deutet vieles darauf hin, dass im fachbezogenen Fremdsprachenunterricht noch stärker als in gemeinsprachlichen Kursen die Lerner aktiv in die Unterrichtsgestaltung mit einbezogen werden müssen, um ihren individuellen Kommunikationsbedürfnissen gerecht zu werden. Ein Lehrwerk kann hierbei immer nur Anhaltspunkte liefern, die im Einzelfall zu überprüfen und gegebenenfalls zu ergänzen sind.

\section{Literaturverzeichnis:}

BECKER, Andrea / HundT, Markus. Die Fachsprache in der einzelsprachlichen Differenzierung. In: HOFFMANN, Lothar / KALVERKÄMPER, Hartmut / WIEGAND, Herbert Ernst (Hg.). Fachsprachen. Languages for Special Purposes. Ein internationales Handbuch zur Fachsprachenforschung und Terminologiewissenschaft. An international Handbook of Special-Language and Terminology Research. Berlin/New York, de Gruyter, 1998, 118-133

BuHLMAnN, Rosemarie. Analyse und Beurteilung fachsprachlicher Lehrwerke: Kriterien und ihre Problematik. In: KRUMM, Hans-Jürgen (Hg.). Lehrwerkforschung - Lehrwerkkritik. Deutsch als Fremdsprache. Protokoll eines Werkstattgesprächs der Fachgruppe Deutsch als Fremdsprache (Fachverband Moderne Fremdsprache) in Zusammenarbeit mit dem Goethe-

\footnotetext{
${ }^{10}$ Ein Beispiel für diese Tendenz ist das Lehrwerk „Alltag, Beruf \& Co.“, 2009 im Hueber-Verlag erschienen, in welchem parallel (und zum Teil kontrastiv) alltags- und berufsrelevante Themen und Kommunikationssituationen vermittelt werden.
} 
Institut in München vom 23. bis 24. Oktober 1981. München, Kemmler \& Hoch, 1982, 122-164

Buhlmann, Rosemarie. Fachsprachliche Lehrmittelsysteme. In: Hoffmann, Lothar / KALVERKÄMPER, Hartmut / WiegAnd, Herbert Ernst (Hg.). Fachsprachen. Languages for Special Purposes. Ein internationales Handbuch zur Fachsprachenforschung und Terminologiewissenschaft. An international Handbook of Special-Language and Terminology Research. Berlin/New York, de Gruyter, 1998, 982-988

FLUCK, Hans-Rüdiger. Didaktik der Fachsprachen. Aufgaben und Arbeitsfelder, Konzepte und Perspektiven im Sprachbereich Deutsch. Tübingen, Narr, 1992

FLUCK, Hans-Rüdiger. Bedarf, Ziele und Gegenstände fachsprachlicher Ausbildung. In: HoffMAnN, Lothar / KALVERKÄMPER, Hartmut / WiEgAnd, Herbert Ernst (Hg.). Fachsprachen. Languages for Special Purposes. Ein internationales Handbuch zur Fachsprachenforschung und Terminologiewissenschaft. An international Handbook of Special-Language and Terminology Research. Berlin/New York, de Gruyter, 1998, 944-954

FunK, Hermann. Berufsbezogener Deutschunterricht - Deutsch als Fremd- und Zweitsprache für den Beruf. In: HeLbiG, Gerhard / GöTzE, Lutz / HenRicI, Gerd / KRUMM, Hans-Jürgen (Hg.). Deutsch als Fremdsprache. Ein internationales Handbuch. Berlin/New York, de Gruyter, 2001, 962-973

Hoffmann, Lothar. Kommunikationsmittel Fachsprache. Eine Einführung. Tübingen, Narr, 1985

HofFMAnN, Lothar. Fachsprachen und Gemeinsprache. In: HofFMANN, Lothar / KALVERKÄMPER, Hartmut / WiEgAND, Herbert Ernst (Hg.). Fachsprachen. Languages for Special Purposes. Ein internationales Handbuch zur Fachsprachenforschung und Terminologiewissenschaft. An international Handbook of Special-Language and Terminology Research. Berlin/New York, de Gruyter, 1998, 157-168

Hoffmann, Lothar. Fachsprachen. In: Helbig, Gerhard / GöTze, Lutz / HenRici, Gerd / KrumM, Hans-Jürgen (Hg.). Deutsch als Fremdsprache. Ein internationales Handbuch. Berlin/New York, de Gruyter, 2001, 533-543

HofFMAnN, Lothar / KALVERKÄMPER, Hartmut / WiEgAnd, Herbert Ernst (Hg.). Fachsprachen. Languages for Special Purposes. Ein internationales Handbuch zur Fachsprachenforschung und Terminologiewissenschaft. An international Handbook of Special-Language and Terminology Research. Berlin/New York, de Gruyter, 1998 